\title{
A New Skin Viewer and Analyzer on Mobile Phone
}

\author{
S Mardanisamani ${ }^{1}$; M Yazdi $^{2,}{ }^{, *}$; J Jamshidzadeh ${ }^{3}$ \\ 'Signal and Image Processing Lab, Department of Electrical and Computer Engineering, Shiraz University, Shiraz, Iran \\ ${ }_{2}^{2}$ Professor, Signal and Image Processing Lab, Faculty of Electrical and Computer Engineering, Shiraz University, Shiraz, Iran \\ ${ }^{3}$ Professor, Faculty of Pharmacy, Shiraz University of Medical Science, Shiraz, Iran \\ * Corresponding author: M Yazdi, Professor, Signal and Image Processing Lab, Faculty of Electrical and Computer Engineering, Shiraz University, Shiraz, Iran, \\ E-mail: yazdi.me@gmail.com
}

Received: 11 Dec 2016

Accepted: 01 Jan 2017

Epub: 23 Feb 2017

Ppub: 15 Jan 2018

\begin{abstract}
Background:Skin image processing on smart phones has become one of the striking and serious research areas in the past few years. A large number of people cannot benefit from the quality care that they need. Mobile application technology offers ways to help with these challenges. An application (App) called UMSkinCheck was reported in 2012, which provides guidance on how to check for skin lesions and moles and also includes information on skin cancer prevention. Last year, another skin cancer App called mole detective was reported. This App gets pictures of skin moles and analyzes them and also calculates a person's risk of skin cancer based on the characteristics of their mole undertaken by dermatologists. In 2016, the SkinVision App, which claims to assist in the early detection of melanoma, was created. This App uses a mathematical theory to analyze photos of skin lesions and moles taken by the user.

objectives: We propose an automatic method for segmenting the skin lesions and extracting features that are associated to them as well as detection skin disorders. In the suggesting step, at first, the region of skin lesion is segmented from the whole skin image; next, some features like the mean, variance, RGB, and HSV parameters are extracted from the segmented region and then by using a classifier to detect skin disorders. We integrate these steps into a mobile App for primary processing and analysis of skin images anywhere and anytime.

Methods: Apps on mobile phones have wide applications in different scientific fields including medicine. By use of image processing algorithms and Java programming, physicians have been more successful in the diagnosis of different skin diseases and have achieved much better treatment results.

Results: Skin viewer and analyzer App can include some benefit features: such as monitoring, tracking, and understanding individuals' skin health, having medical image processing operations, as well as allows the individuals to capture all of their moles and skin conditions to thoroughly understand their skin.

Conclusion: This important technology provides physicians with the ability to immediately view the skin and make a diagnosis. Skin Apps offer a unique technology to detect early and potential signs of lesion disorder and skin cancer growth.
\end{abstract}

Keywords: mHealth; Skin Disorder; Mobile App 